\title{
Actividad Biocida del Aceite Esencial de Lippia origanoides H.B.K sobre Phytophthora infestans (Mont.) de Bary
}

\author{
Julian M. Acosta(1,2), Oscar Arango(2), David E. Álvarez ${ }^{(2)}$ y Andrés M. Hurtado(2) \\ (1) Grupo GIIDOP, Facultad de Ingeniería, Programa Ingeniería de Procesos, Universidad Mariana, Calle 18 No. 34-104, \\ Pasto-Nariño, Colombia (e-mail: jacosta@umariana.edu.co) \\ (2) Grupo TEA, Facultad de Ingeniería Agroindustrial, Universidad de Nariño, Carrera 33 No. 5-121, Pasto-Nariño, \\ Colombia. (oscar.arangob@gmail.com; ingdeas@hotmail.com; ahurtadob@hotmail.com)
}

Recibido Ene. 3, 2019; Aceptado Feb. 28, 2019; Versión final Jun. 21, 2019, Publicado Dic. 2019

\section{Resumen}

Se evaluó el aceite esencial de Lippia origanoides H.B.K. como alternativa para el control del fitopatógeno $P$. infestans aislado de tomate Solanum lycopersicum L. Para ello, se extrajo el aceite esencial por arrastre con vapor, identificando y cuantificando la composición por GC-MS. A nivel in vitro se calculó la acción biocida mediante la técnica de difusión en agar estimando la concentración mínima inhibitoria y por bioautografía se registró la fracción activa. Los resultados identificaron a timol (83\%) como el compuesto mayoritario del aceite. Los ensayos in vitro mostraron alta sensibilidad del fitopatógeno con una concentración mínima inhibitoria de $75 \mu \mathrm{L} \cdot \mathrm{mL}^{-1}$ en donde el timol causó la actividad biocida. Con estos resultados se demuestra el potencial del aceite esencial para el manejo del fitopatógeno y su viabilidad para el desarrollo de un bioinsumo agrícola.

\section{Biocidal Activity of the Essential Oil of Lippia origanoides H.B.K on Phytophthora infestans (Mont.) of Bary}

\begin{abstract}
The essential oil of wild Lippia origanoides H.B.K was evaluated as an alternative for the control of the phytopathogen P. infestans isolated from tomato Solanum lycopersicum L. To do this, the essential oil was extracted by steam entrainment, identifying and quantifying the composition by GC-MS. At in vitro level, the biocidal action was calculated using the agar diffusion technique, estimating the minimum inhibitory concentration and the active fraction was recorded by bioautography. The analyses identified thymol ( $83 \%)$ as the major compound in the oil. The in vitro tests showed high sensitivity of the phytopathogen with a minimum inhibitory concentration of $75 \mu \mathrm{L} \cdot \mathrm{mL}^{-1}$ where the thymol caused the biocidal activity. With these results, the potential of the essential oil for the management of the phytopathogen and its viability for the development of an agricultural bio consumables.
\end{abstract}

Keywords: Solanum lycopersicum L; wild oregano; thymol; bioautography; bio consumables 


\section{INTRODUCCIÓN}

A nivel mundial el tomate de mesa Solanum lycopersicum L. se cataloga como una de las hortalizas más importantes debido a sus variadas aplicaciones y usos como fruto en fresco y producto procesado, de allí que el cultivo se ha intensificado en gran medida en los últimos años. No obstante, el modelo de producción presenta diversos problemas fitosanitarios entre los cuales se destaca el "tizón tardío" causado por Phytophthora infestans (Mont.) de Bary, generando pérdidas entre el 70 y $100 \%$ de la cosecha cuando no se realiza un manejo técnico adecuado (Martínez et al., 2016). Actualmente la medida de protección y supresión de $P$. infestans en campo se limita a la aplicación calendarizada de plaguicidas sintéticos. Por ello, es imperante la necesidad de buscar estrategias sostenibles frente a este problema, que permita reducir los efectos negativos generados sobre el ambiente y el hombre (Ahmad et al., 2011).

Una de las alternativas más prometedoras es el empleo de productos naturales extraídos de plantas aromáticas, como es el caso de los aceites esenciales (A.E.). Estos, se han integrado a las medidas de manejo en la agricultura debido al efecto biocida que tienen los compuestos que hacen parte de este metabolito secundario de las plantas. Los A.E. son mezclas homogéneas de compuestos químicos orgánicos oxigenados como alcoholes, acetonas, cetonas, éteres y aldehídos comúnmente concentradas en los canales secretores de hojas, corteza, frutos y flores de plantas aromáticas. Estos por lo general, se clasifican según sus componentes mayoritarios en monoterpenoides, sesquiterpenoides y fenilpropanoides (García et al., 2010; Torrenegra et al., 2015).

En tal razón, varios investigadores indican el alto potencial del A.E. extraído de orégano silvestre ( $L$. origanoides) un arbusto de la familia Verbenaceae, para el manejo de problemas fitosanitarios en agricultura orgánica (Arango et al., 2014, Álvarez et al., 2016). En este A.E., se reporta la presencia aproximada de 54 compuestos entre los que se destaca el timol, carvacrol y $\rho$-cimeno a los cuales se les atribuyen actividad antioxidante, antigenotoxica, antoprotozoica, antifungica y antibacterial (Bueno et al., 2011; Escobar et al., 2010; Muñoz-Acevedo et al., 2009; Vicuña et al., 2010). Por lo anterior, en este trabajo se buscó evaluar la actividad biocida del A.E. de orégano silvestre sobre el fitopatógeno $P$. infestans aislado de tomate de mesa, con el fin de establecer la viabilidad para integrarlo a un plan de manejo limpio de la enfermedad del "tizón tardío".

\section{METOdOLOGÍA}

La metodología se describe en cuatro subsecciones para mayor claridad de la presentación: (i) obtención del A.E., (ii) fraccionamiento y análisis de la composición del A.E., (iii) evaluación del A.E. sobre $P$. infestans, ensayo de difusión, y (iv) evaluación del A.E. sobre $P$. infestans, ensayo de bioautografía

\section{Obtención del aceite esencial (A.E.)}

El material vegetal usado para la extracción de A.E. estuvo constituido por hojas de orégano silvestre recolectadas en la vereda Alto del Mayo, ubicada en el área subxerofitica denominada Cordón Panamericano del Alto Patía, entre los municipios de Taminango norte del departamento de Nariño y Mercaderes sur del departamento del Cauca (Colombia), a una altura de $1.200 \mathrm{msnm}$. La recolección se realizó en época de lluvias, en los dos tercios superiores del arbusto en floración. Una vez obtenidas las hojas se secaron bajo sombra sobre una superficie de cemento por dos días, hasta alcanzar una humedad aproximada del $14 \%$. El proceso de extracción del A.E. se realizó en la Planta Piloto de la Facultad de Ingeniería Agroindustrial, localizada en el municipio de San Juan de Pasto (Nariño), a $2.527 \mathrm{msnm}$ utilizando la técnica de arrastre con vapor. Para ello se utilizó de un equipo de extracción de 80 litros, dos condensadores en serie, uno vertical y otro horizontal acoplado a un sistema de separación del A.E. mediante vaso florentino. Se realizaron 5 extracciones cada una con $7 \mathrm{Kg}$ de material vegetal, en las siguientes condiciones: tiempo 1,5 horas, presión 1 psig y temperatura $94^{\circ} \mathrm{C}$ (Arango et al., 2014; Arango et al., 2012), seguido de un ciclo de rotaevaporación (EYELA® oil bath osb-2000) con el fin de eliminar impurezas y agua residual. El aceite obtenido se depositó en envases de vidrio ámbar y almacenó en nevera a $5^{\circ} \mathrm{C}$ hasta su caracterización y evaluación.

\section{Fraccionamiento y análisis de la composición del A.E.}

El fraccionamiento del A.E. se obtuvo aplicando $1 \mu \mathrm{l}$ de aceite por placa de Cromatografía en Capa Delgada (C.C.D.) preparativa de $20 \times 20 \mathrm{~cm}$, con fase estacionaria de $1 \mathrm{~mm}$ de sílica-gel $60 \mathrm{GF}_{254}$ utilizando como fase móvil la mezcla de tolueno/acetato de etilo en relación 9:1 (v/v). Las fracciones fueron visualizadas con luz ultravioleta con longitud de onda de $254 \mathrm{~nm}$ y a través de revelado con solución de vanilina sulfúrica.

Para cada fracción desagregada en la placa de cromatografía se calculó el factor de retención Rf utilizando la ecuación 1; dónde $\mathrm{A}$ es la distancia recorrida por el compuesto y B es la distancia recorrida por la mezcla de solventes (Álvarez et al., 2016). 


$$
R f=\frac{(A)}{(B)}
$$

Para la caracterización del A.E. y las fracciones obtenidas mediante C.C.D. se sometieron a un análisis composicional utilizando un cromatógrafo de gases con detector selectivo de masas (GC-MS) marca Shimadzu (Japón), modelo QP2010S, con columna capilar SHRIX-5MS (30 m x 0,25 mm ID x 0,25 $\mu \mathrm{m}$ ), con inyección a $250^{\circ} \mathrm{C}$ modo Split 1:50, volumen de inyección: $1,0 \mu \mathrm{L}$, (Stashenko y Martínez 2010). La identificación de los compuestos se realizó utilizando los índices de retención de kovats (Ik) usando n-alcanos $\left(\mathrm{C}_{6}-\mathrm{C}_{32}\right)$, los espectros de masas obtenidos se compararon con los establecidos en la librería de espectros Wiley®. La cuantificación se realizó mediante el cálculo del porcentaje relativo de áreas de cada compuesto.

\section{Evaluación del A.E. sobre $P$. infestans, ensayo de difusión}

Se realizó el aislamiento del fitopatógeno, tomando muestras de hojas de tomate de mesa visiblemente afectadas con $P$. infestans, las cuales se cortaron en pedazos de $5 \mathrm{~mm} \times 5 \mathrm{~mm}$, posteriormente se lavaron con una solución de hipoclorito de sodio al $0,5 \%$ y agua destilada. Se procedió a su siembra en cajas Petri con medio V8 suplementado con una solución de Rifampicina, las cajas se llevaron a la cámara de incubación a temperatura de $18^{\circ} \mathrm{C}$. Después de 120 horas se procedió a purificar el fitopatógeno teniendo en cuenta características macroscópicas de las colonias y luego la identificación de rasgos morfológicos como micelio cenocítico, producción de esporangios limoniformes, esporangióforos con crecimiento indeterminado y otras propias de los Oomycetes. Para el montaje del bioensayo, el A.E de L. origanoides se emulsionó con Tween $20(1: 1 \mathrm{v} / \mathrm{v})$. Esta mezcla se adicionó al medio de cultivo V8 de acuerdo a las concentraciones a evaluar; luego, el medio se sirvió en cajas Petri y se inoculó el fitopatógeno con un disco de micelio de $1,1 \mathrm{~cm}$ el mismo que se colocó en el centro de la caja. El montaje fue incubado en oscuridad a una temperatura de $18^{\circ} \mathrm{C}$ durante 192 horas. Para la evaluación del fitopatógeno se utilizó un diseño completamente al azar (D.I.A.) con seis tratamientos: $10,100,1.000$ y $10.000 \mu \mathrm{L} . \mathrm{mL}^{-1}$ de A.E., un testigo con fungicida sintético i.a. Metalaxil $4 \%$ (2 $\left.\mathrm{mg} \mathrm{mL}^{-1}\right)$ y un testigo absoluto. Cada tratamiento contó con 15 repeticiones, el ensayo se replicó dos veces en el tiempo siguiendo la metodología usada por Álvarez et al. (2016).

\section{Variables respuesta}

El porcentaje de crecimiento (PC) se determinó a las 192 horas mediante la medición del diámetro micelial y transformación a términos porcentuales, usando la ecuación 2 propuesta por Riveros et al. (2003); dónde DMCM es el diámetro medio colonia creciendo en tratamiento, $1,1 \mathrm{~cm}$ es el diámetro del cilindro con micelio y DMCA hace referencia al diámetro de crecimiento del testigo.

$$
P C=\left(\frac{D M C M-1,1}{D M C A}\right) * 100
$$

La sensibilidad se estimó mediante la escala propuesta por Shattock (1998), en donde, sensibles (S): crecimiento < $10 \%$ del testigo; Intermedio (I): crecimiento entre 10 y $60 \%$ del testigo y Resistente $(R)$ : crecimiento $>60 \%$ del testigo. La concentración media efectiva cincuenta $\left(\mathrm{CE}_{50}\right)$ se estimó mediante un análisis Probit que determinó las concentraciones efectivas tanto para el A.E. como para el fungicida empleado en el estudio. Para ello, se relacionó el valor del crecimiento micelial a las 192 horas con el testigo absoluto, transformando los valores a términos porcentuales y calculando el valor de crecimiento $50 \%$ (Álvarez et al., 2016). Para identificar diferencias entre tratamientos se utilizó la técnica de análisis de varianza ANDEVA $(\alpha=0,05)$ y la prueba de comparación de medias de Tukey $(\alpha=0,05)$, usando el programa estadístico InfoStat v. 2018.

\section{Evaluación del A.E. sobre $P$. infestans, ensayo de bioautografía}

Sobre las placas cromatográficas donde previamente fue separado el A.E. se dispuso una capa fina de medio de cultivo V8 y se inoculó una solución de agua destilada y esporangios de $P$. infestans obtenidos de cultivos puros $\left( \pm 1 \times 10^{6}\right.$ esporangios. $\left.\mathrm{ml}^{-1}\right)$. Las placas se dispusieron en cajas de Petri y se incubó a $20^{\circ} \mathrm{C}$ por un período de 192 horas. En las regiones donde el microorganismo no se desarrolló correspondió a la fracción activa del A.E., esa fracción se identificó en otras placas por comparación entre el factor de retención y el largo de la banda. Para recuperar la fracción activa, se procedió a raspar el área correspondiente en visualización UV de cada placa, posteriormente se extrajo con diclorometano y agitación magnética por 3 horas, analizando luego su composición mediante GS-MS (Sridhar et al., 2003). 


\section{RESULTADOS Y DISCUSIÓN}

Los resultados obtenidos en esta investigación se detallan en seis subsecciones: (i) Obtención del A.E., (ii) Fraccionamiento y análisis de la composición del A.E., (iii) Evaluación del A.E. sobre P. infestans, ensayo de difusión, (iv) Concentración Mínima Inhibitoria (CMI), (v) Concentración efectiva 50 (CE50), y (vi) Evaluación del A.E. sobre $P$. infestans, ensayo de bioautografía.

\section{Obtención del aceite esencial (A.E.)}

Se obtuvo un rendimiento de $2,81 \%$ calculado a partir de la cantidad de A.E. extraído por unidad de masa seca de materia prima, que de acuerdo con la clasificación presentada por Kintzios (2002) puede identificarse este valor como "rich" o abundante para una especie vegetal. El rendimiento de A.E. de orégano silvestre fue superior que el reportado por Staschenko et al. (2014) para las especies de la familia Verbenace como Lippia alba, L. citriodora, L. micromera, L. americana, L. graveolens y L. dulcis, los cuales solamente alcanzan rendimientos entre 0,1 y $1,2 \%$, indicando que la especie estudiada en Nariño presenta potencial industrial por su alto contenido en aceite.

En cuanto a la comparación dentro de la especie, para $L$. origanoides se encontraron reportes que indican una variación en el rendimiento entre 1,5 a 4,6\%, destacándose las condiciones ambientales en las cuales se desarrolla el arbusto y la técnica de extracción para explicar los resultados obtenidos. En este sentido, Torrenegra et al. (2015) indican que el clima, altitud, tipo de suelo, luminosidad, pluviosidad, temperatura, entre otros, generan diversidad de ecosistemas y microclimas, lo cual hace que la especie vegetal presente variaciones en el contenido de metabolitos secundarios. Por ejemplo, Arango et al. (2012) demuestran variación en el contenido de aceite por efecto de las condiciones climáticas en el año, presentando mayores rendimientos de A.E. en época seca; también, Staschenko et al. (2014) y Álvarez et al. (2016) reportan efecto de la localidad en el contenido de A.E. de cinco muestras de L. origanoides colectadas en Colombia (departamentos de Nariño, Cauca y Santander).

Por otra parte, el método de extracción también genera diferencias en el rendimiento de A.E., siendo el más reportado el arrastre con vapor con variaciones en tiempo de extracción, presión y densidad de lecho, presentando un rendimiento promedio de 3,08 \%, aproximado al obtenido en este estudio (Arango et al., 2012; Vásquez, 2012; Arango et al., 2014). También se reporta en bibliografía el uso del método de hidrodestilación asistida por la radiación de microondas, presentando un mayor valor promedio (4,5\%) como indican Staschenko et al. (2014), Stashenko y Martínez, (2010) y Escobar et al. (2010), esta técnica podría mejorar la eficiencia de extracción de A.E. en esta especie.

\section{Fraccionamiento y análisis de la composición del A.E.}

En la Figura 1. se muestra la placa cromatográfica y la separación de cuatro fracciones del A.E., en este montaje se calculó el factor de retención (Rf) y se identificó a partir de GC-MS los compuestos presentes en cada fracción como se detalla en la Tabla 1. Empleando la misma metodología, se realizó una corrida con un patrón comercial de timol al 99,9\% de pureza (T0501 SIGMA®), los resultados demostraron que este monoterpeno presentó un factor de retención $R f=0,62$ igual al establecido en la fracción $3\left(F_{3}\right)$.

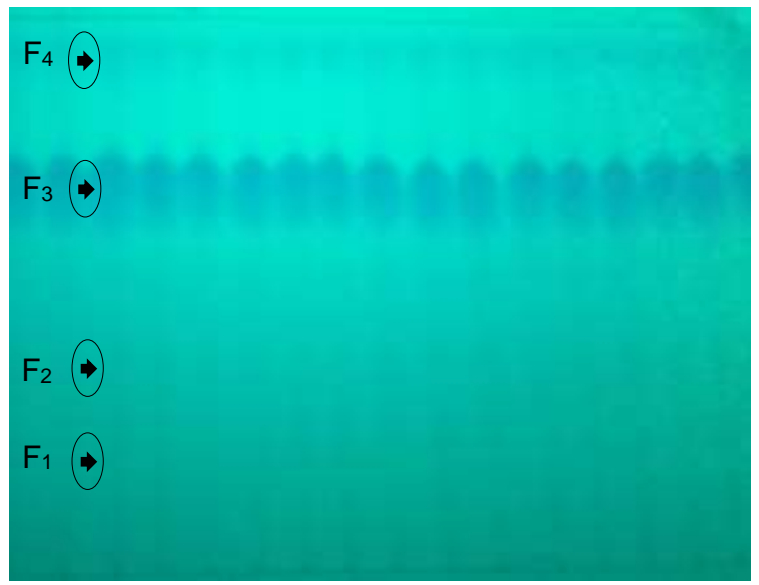

Fig. 1: Fracciones del A.E. de L. origanoides en Cromatografía en Capa Delgada 
Tabla 1: Principales compuestos identificados en las fracciones de A.E. de L. origanoides.

\begin{tabular}{|c|c|c|c|}
\hline Fracción & Rf (Factor de retención) & Identificación por GC-FID & Tiempo de retención $\mathrm{Tr}$ \\
\hline Fracción 1 (F1) & 0,14 & $\begin{array}{c}\text { Timilo } \\
\text { Acetato de carvacrilo }\end{array}$ & $\begin{array}{l}20,09 \\
30,11\end{array}$ \\
\hline Fracción 2 (F2) & 0,35 & $\begin{array}{c}\text { Carvacrol } \\
\beta \text {-Elemeno }\end{array}$ & $\begin{array}{l}27,30 \\
30,50\end{array}$ \\
\hline Fracción 3 (F3) & 0,62 & Timol & 25,15 \\
\hline Fracción 4 (F4) & 0,67 & $\begin{array}{l}\text { Y-Muruleno } \\
\text { Timol }\end{array}$ & $\begin{array}{l}32,95 \\
25,15\end{array}$ \\
\hline
\end{tabular}

De forma cualitativa se pudo determinar en la placa cromatográfica que la fracción $3\left(F_{3}\right)$ en todos los ensayos presentó un mayor tamaño al visualizarla por UV, el mismo resultado lo obtuvo Vásquez (2012) quien realizó el fraccionamiento del mismo A.E. utilizando la técnica de cromatografía de columna flash (C.C.F.) y C.C.D. con fase móvil pentano y éter dietílico, encontrando que la fracción que contenía timol en los dos casos presentó la mayor área, concordando más adelante con ensayos cuantitativos; de acuerdo a las condiciones del estudio de Vásquez (2012) se reporta un Rf de 0,30 y 0,40 respectivamente.

Por otro lado, la identificación analítica del aceite por GC-FID/MS en la Tabla 2 y Figura 2 reportó 16 compuestos en base a los patrones de fragmentación y tiempo de retención, número que dista de los reportes de Staschenko et al. (2014) quienes indican un promedio de 33 compuestos identificados mediante la misma técnica cromatográfica, posiblemente las condiciones de montaje experimental y la escala de evaluación expliquen la diferencia presentada. Sin embargo, es importante destacar que la mayoría de estudios sobre A.E. se basan en los compuestos con un porcentaje relativo superior a $1 \%$ de la fracción, bajo esta premisa, en esta investigación se destaca la presencia de timol (83\%), $p$-cimeno $(5,5 \%)$, mirceno (3\%), $\gamma$-terpineno $(2,5 \%)$ y carvacrol $(1 \%)$.

Estos resultados son similares a los encontrados por Betancourt et al. (2012), quienes reportaron para el A.E. de $L$. origanoides recolectado en la misma zona de muestreo de este estudio (vereda Alto del Mayo) como principales constituyentes al timol $(78,7 \%)$, p-cimeno $(6,6 \%)$, $\mathrm{y}$-terpineno $(2,7 \%)$ y carvacrol $(0,9 \%)$, por otro lado, Do Santos et al., (2004), reportan composiciones diferentes donde identificaron a monoterpenos como carvacrol $(38,2 \%)$, y-terpineno $(9,3 \%)$, timol $(6,8 \%)$, metil timol $(7,4 \%)$ y $p$-cimeno $(13,9 \%)$ como principales constituyentes, finalmente se cita el reporte de Staschenko et al. (2014) para la zona de Nariño identificando timol $(54,5 \%)$, $y$-terpineno $(5,0 \%)$ y acetato de timilo $(4,8 \%)$.

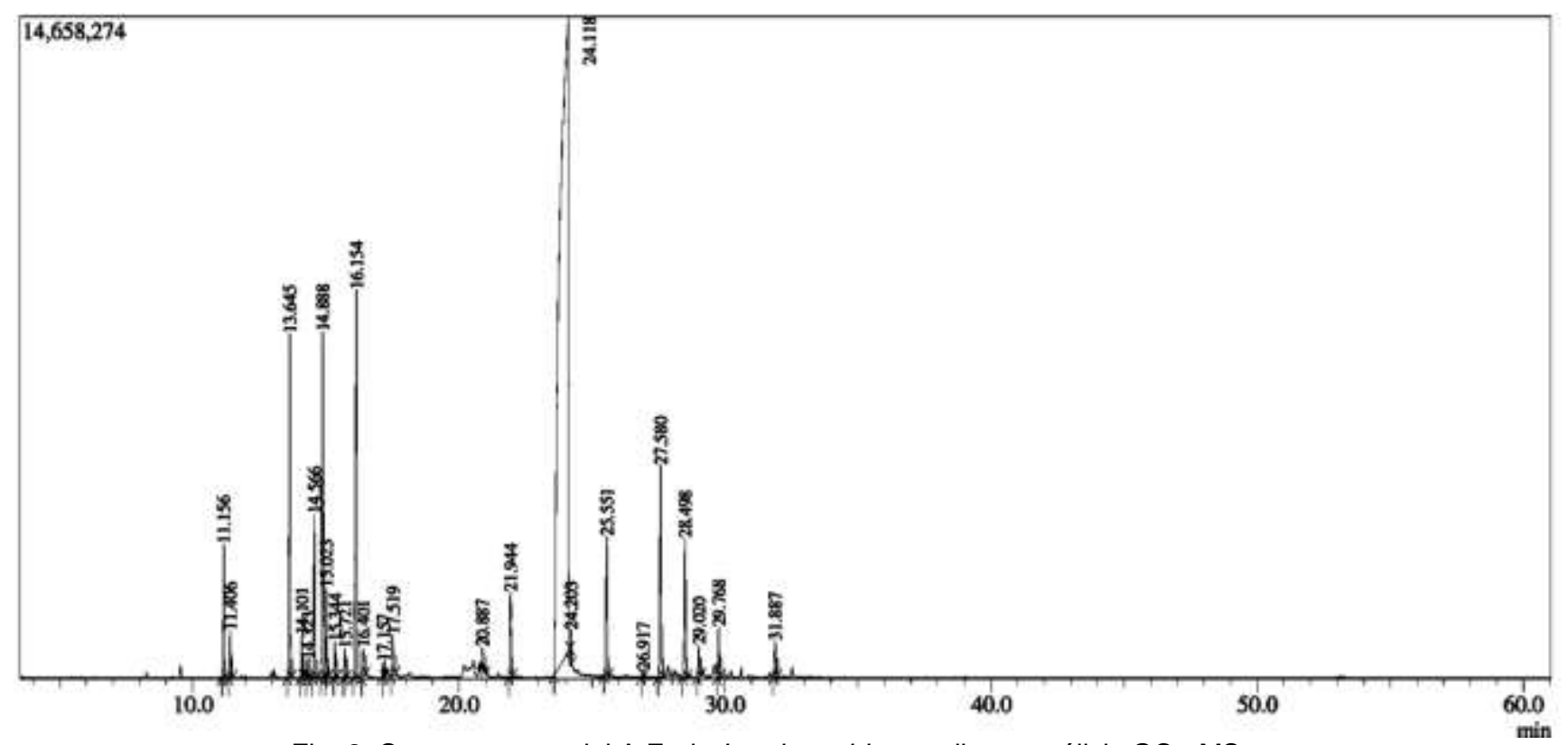

Fig. 2: Cromatograma del A.E. de L. origanoides mediante análisis GC - MS.

Las variaciones en la composición química de metabolitos secundarios en plantas de la misma especie han permitido designar diferentes quimiotipos. Además de este factor, Arango et al. (2012) no solo evaluó los cambios en el de A.E. por condiciones ambientales y fenológicas del arbusto de orégano silvestre, si no que indicaron variaciones en el contenido de timol en el A.E., siendo la época seca el mejor momento para obtención del monoterpeno incrementando aproximadamente en 15,4 \% el contenido relativo en relación a época lluviosa. De igual forma, Dos Santos et al. (2004) y Stashenko y Martínez (2010) afirman que los 
compuestos del aceite esencial de orégano se ven afectados por las condiciones edafológicas y ambientales experimentadas durante el crecimiento de la planta, entre ellas las características del suelo, duración de la luz solar, temperatura y estrés de agua.

Tabla 2: Principales compuestos identificados en el A.E. de L. origanoides.

\begin{tabular}{|c|c|c|c|l|}
\hline No. & $\begin{array}{c}\text { Tiempo de } \\
\text { retención (tR) }\end{array}$ & $\begin{array}{c}\text { Cantidad } \\
\text { Relativa (\%) }\end{array}$ & IRL & Identificación \\
\hline 1 & 12,28 & 0,5 & 941 & $\alpha$-Pineno \\
\hline 2 & 12,54 & 0,2 & 946 & Canfeno \\
\hline 3 & 14,92 & 3,0 & 999 & Mirceno \\
\hline 4 & 15,47 & 0,2 & 1.012 & $\alpha$-Felandreno \\
\hline 5 & 15,87 & 0,7 & 1.021 & $\alpha$-Terpineno \\
\hline 6 & 16,46 & 5,5 & 1.035 & $\rho$-Cimeno \\
\hline 7 & 17,51 & 2,5 & 1.059 & -Terpineno \\
\hline 8 & 23,04 & 0,3 & 1.193 & Terpinen-4-ol \\
\hline 9 & 26,74 & 83,0 & 1.294 & Timol \\
\hline 10 & 27,29 & 1,0 & 1.309 & Carvacrol \\
\hline 11 & 29,02 & 1,0 & 1.357 & Acetato de Timilo \\
\hline 12 & 29,93 & 0,7 & 1.382 & Acetato de Carvacrilo \\
\hline 13 & 30,53 & 0,3 & 1.398 & $\beta$-Elemeno \\
\hline 14 & 31,08 & 0,3 & 1.414 & $\beta$-Cariofileno \\
\hline 15 & 33,21 & 0,4 & 1.474 & $\gamma$-Muruleno \\
\hline 16 & 33,96 & 0,2 & 1.495 & $\beta$-Bisaboleno \\
\hline
\end{tabular}

Los resultados encontrados en esta investigación establecen que el A.E. de orégano silvestre del Alto Patía contiene cantidades más elevadas de timol frente a las encontradas en la misma especie establecida de diferentes regiones de Colombia y de otras especies el género Lippia en Suramérica (Staschenko et al., 2014), mostrando un alto potencial agroindustrial que debería explorarse más a fondo.

\section{Evaluación del A.E. sobre P. infestans, ensayo de difusión}

Para el análisis de la sensibilidad del fitopatógeno frente a los compuestos presentes en el A.E. de orégano silvestre, se realizaron dos ensayos; el primer ensayo permitió identificar los rangos de concentraciones de aceite esencial que afectan el crecimiento del patógeno evaluando de forma exponencial un rango entre $10 \mathrm{y}$ $10.000 \mu \mathrm{g} \mathrm{mL}^{-1}$. Esta evaluación se realizó en un periodo de $192 \mathrm{~h}$, en este periodo el testigo absoluto T0, presentó un crecimiento promedio de $86,7 \%$ presentando diferencias estadísticas respecto a todas las concentraciones del A.E. evaluadas, se destacan los tratamientos T1 y T2 con un crecimiento de 5,0 y $1,1 \mathrm{~cm}$ respectivamente, a partir de T3 se inhibe totalmente el crecimiento del Oomyceto a nivel de laboratorio, los resultados de esta evaluación se presentan en la figura 3, y Tabla 3.

Tabla 3: Resultados de Ensayo 1, actividad in vitro del A.E. frente a $P$. infestans.

\begin{tabular}{|c|c|c|c|c|c|}
\hline \multirow{2}{*}{ Tratamiento } & \multirow{2}{*}{$\begin{array}{c}\text { Concentración } \\
\left(\mu g \mathrm{~mL}^{-1}\right)\end{array}$} & 48 & 96 & \multicolumn{4}{|c|}{ Tiempo de evaluación $(\mathrm{h})$} \\
\cline { 3 - 6 } & 0 & $31,6^{\mathrm{a}}$ & $48,5^{\mathrm{a}}$ & $80,9^{\mathrm{a}}$ & $86,7^{\mathrm{a}}$ \\
\hline T0 & 10 & $12,4^{\mathrm{b}}$ & $16,4^{\mathrm{b}}$ & $29,1^{\mathrm{b}}$ & $46,9^{\mathrm{b}}$ \\
\hline T1 & 100 & $0^{\mathrm{c}}$ & $0^{\mathrm{c}}$ & $0^{\mathrm{c}}$ & $0^{\mathrm{c}}$ \\
\hline T2 & 1.000 & $0^{\mathrm{c}}$ & $0^{\mathrm{c}}$ & $0^{\mathrm{c}}$ & $0^{\mathrm{c}}$ \\
\hline T3 & 10.000 & $0^{\mathrm{c}}$ & $0^{\mathrm{c}}$ & $0^{\mathrm{c}}$ & $0^{\mathrm{c}}$ \\
\hline T4 & \multicolumn{5}{c}{} \\
\hline
\end{tabular}

Los resultados obtenidos en la segunda evaluación (Ensayo 2), determinaron el efecto biocida del A.E. en el nuevo rango de evaluación ( 0 a $100 \mu \mathrm{g} \mathrm{mL}^{-1}$ ), tal como se describen en la Tabla 4 y figura 4 . La figura 4 permite identificar de forma visual el efecto biocida del A.E. a las concentraciones de 50 y $75 \mu \mathrm{g} \mathrm{mL}^{-1}$ frente a $P$. infestans. En las figuras 3 y 4 , se puede evidenciar las diferencias existentes entre las concentraciones aplicadas, en este sentido, el A.E. de L. origanoides del Alto Patía permite solo un crecimiento promedio de $53,26 \%$ bajo una concentración de $10 \mathrm{~mL}^{-1}$ y a una concentración de $100 \mathrm{~mL}^{-1}$ al igual que en el primer ensayo, no se observó crecimiento ratificando el efecto encontrado y establecer la CMI de la evaluación in vitro. A continuación, se presenta el consolidado de los ensayos 1 y 2 y su análisis de acuerdo a la escala de Shattock, los cuales se compilan en la Tabla 5.

La escala Shattock (1998), define tres grupos: el primero resistente con porcentaje de crecimiento mayor a $60 \%$ en donde se encuentra la concentración cero (sin aceite esencial), el segundo intermedio con porcentaje de crecimiento entre $10 \%$ y $60 \%$ en donde se encuentra las concentraciones de 10,20 y $30 \mu \mathrm{g} \cdot \mathrm{mL}^{-1}$ y el tercero sensible con porcentaje de crecimiento menores al 10\% se encuentran las concentraciones desde 50 a $10.000 \mu \mathrm{g} \cdot \mathrm{mL}^{-1}$. 


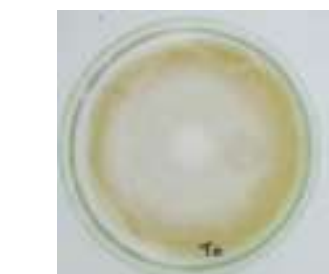

Testigo absoluto $0 \mu \mathrm{g} \cdot \mathrm{mL}^{-1}$

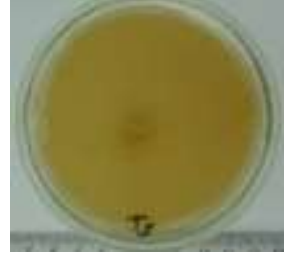

$1.000 \mu \mathrm{g} \cdot \mathrm{mL}^{-1}$

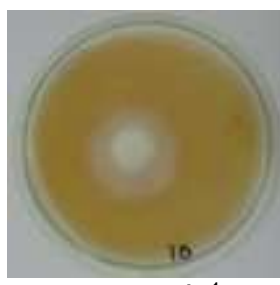

$10 \mu \mathrm{g} \cdot \mathrm{mL}^{-1}$

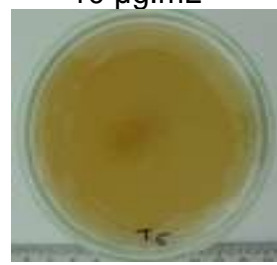

$10.000 \mu \mathrm{g} \cdot \mathrm{mL}^{-1}$

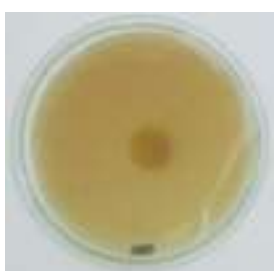

$100 \mu \mathrm{g} \cdot \mathrm{mL}^{-1}$

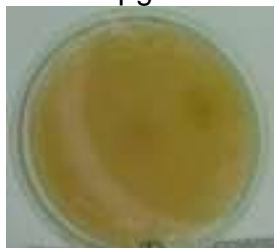

Testigo químico i.a. Metalaxil $2 \mu \mathrm{g} \cdot \mathrm{mL}^{-1}$

Fig. 3: Ensayo 1, crecimiento de $P$. infestans a las 192 horas

Tabla 4: Resultados de Ensayo 2, actividad in vitro del A.E. frente a $P$. infestans.

\begin{tabular}{|c|c|c|c|c|c|}
\hline \multirow[t]{2}{*}{ Tratamiento } & \multirow{2}{*}{$\begin{array}{c}\text { Concentración } \\
\left(\mu g \mathrm{~mL}^{-1}\right)\end{array}$} & \multicolumn{4}{|c|}{ Tiempo de evaluación (h) / Crecimiento (\%) } \\
\hline & & 48 & 96 & 144 & 192 \\
\hline T0 & 0 & $35,3^{a}$ & $54,2^{a}$ & $81,7^{a}$ & $87,2^{a}$ \\
\hline T1 & 10 & $5,9^{b}$ & $20,8^{b}$ & $33,3^{b}$ & $45,9^{b}$ \\
\hline T2 & 20 & $5,9^{b}$ & $12,5^{\mathrm{bc}}$ & $28,3^{c}$ & $28,2^{c}$ \\
\hline T3 & 30 & $0^{\mathrm{b}}$ & $8,3^{\mathrm{bc}}$ & $16,7^{d}$ & $23,5^{c}$ \\
\hline T4 & 50 & $0^{\mathrm{b}}$ & $4,2^{\mathrm{c}}$ & $3,3^{e}$ & $5,6^{d}$ \\
\hline T5 & 75 & $0^{b}$ & $0^{c}$ & $0^{\mathrm{e}}$ & $0^{\mathrm{e}}$ \\
\hline T6 & 100 & $0^{b}$ & $0^{c}$ & $0^{e}$ & $0^{e}$ \\
\hline
\end{tabular}

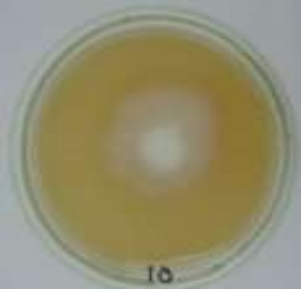

$10 \mu \mathrm{g} / \mathrm{mL}$

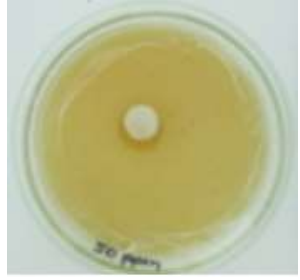

$50 \mu \mathrm{g} / \mathrm{mL}$

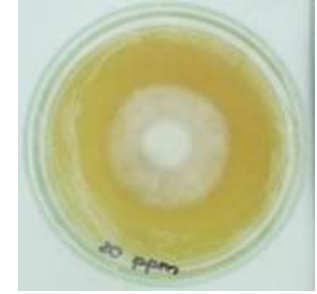

$20 \mu \mathrm{g} / \mathrm{mL}$

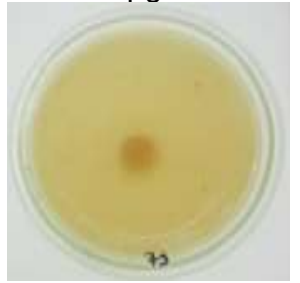

$75 \mu \mathrm{g} / \mathrm{mL}$

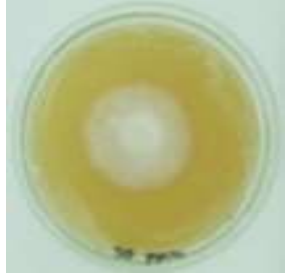

$30 \mu \mathrm{g} / \mathrm{mL}$

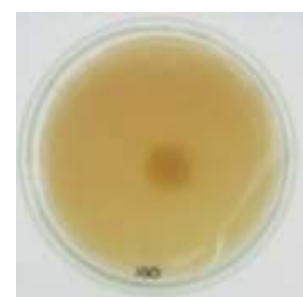

$100 \mu \mathrm{g} / \mathrm{mL}$

Fig. 4: Ensayo 2, crecimiento de $P$. infestans a las 192 horas

Tabla 5: Porcentaje de crecimiento de $P$. infestans y clasificación de acuerdo a la escala Shattock.

\begin{tabular}{|c|c|c|}
\hline $\begin{array}{c}\text { Concentración } \\
\mu g . \mathrm{mL}^{-1}\end{array}$ & Crecimiento (\%) & $\begin{array}{c}\text { Escala } \\
\text { Sensibilidad }\end{array}$ \\
\hline 0 & 87,2 & Resistente \\
\hline 10 & 45,9 & Intermedio \\
\hline 20 & 28,2 & Intermedio \\
\hline 30 & 23,5 & Intermedio \\
\hline 50 & 5,6 & Sensible \\
\hline 75 & 0 & Sensible \\
\hline 100 & 0 & Sensible \\
\hline 1.000 & 0 & Sensible \\
\hline 10.000 & 0 & Sensible \\
\hline
\end{tabular}




\section{Concentración Mínima Inhibitoria (CMI)}

Los resultados obtenidos en esta investigación demuestran que al A.E. de orégano silvestre del Alto Patía tiene un efecto inhibidor importante contra el fitopatógeno $P$. infestans, como se muestra en el grafico 2, desde una concentración mínima probada de $10 \mu \mathrm{g} \cdot \mathrm{mL}^{-1}$ se presentó un porcentaje de inhibición de 57,83\% en el crecimiento del patógeno y a partir de una concentración de $75 \mu \mathrm{g} \cdot \mathrm{mL}^{-1}$ se inhibió completamente su crecimiento siendo esta la CMI, por lo cual se considera que es la concentración letal para este ensayo. Estudios similares como el de Ruano y Figueroa (2013) evaluaron la actividad biocida del mismo A.E. sobre el fitopatógeno Rhizoctonia solani J. G. Kühn aislado de papa (Solanum tuberosum L.) encontrando que en $110 \mu \mathrm{g} \mathrm{mL}-1$ (110 ppm) se inhibió completamente el crecimiento del microorganismo. Comparado con los datos de esta investigación se observa que el A.E. de orégano silvestre, tiene un efecto similar sobre este fitopatógeno dado que en esta misma concentración se inhibe totalmente el crecimiento de $P$. infestans aislado de tomate de mesa.

Ruano y Figueroa (2013) afirman que esta fuerte acción antimicrobiana y antifúngica se debe a la presencia de los terpenoides; así mismo, Hernández et al., (2007) han demostrado la actividad fungicida de aceites esenciales que contienen timol sobre hongos fitopatógenos tales como Botrytris cinerea Pers. y Fusarium oxysporum. Trombetta et al., (2005) realizaron estudios referentes al timol, demostrando que este terpenoide tiene la capacidad de interactuar con las membranas fosfolipídicas, alterando la permeabilidad de la membrana del microorganismo, igualmente, Krolow et al. (2006), evaluaron la actividad antifúngica del A.E. de orégano (Origanum vulgare L.) contra el patógeno $P$. infestans encontrando que con $100 \mu \mathrm{g} \mathrm{mL}^{-1}$ se redujo el crecimiento en un 59,84\%. Comparando con los resultados de esta investigación se puede evidenciar que el A.E. de orégano silvestre del Alto Patía, tiene mayor eficacia sobre este patógeno dado que en una concentración más baja $\left(75 \mathrm{~mL}^{-1}\right)$ se logra inhibir completamente el crecimiento del patógeno, tal efecto se le atribuye al alto contenido de timol. De la misma forma Celis et al. (2007), afirman que el A.E. de L. origanoides es rico en compuestos fenólicos como el timol, el cual exhibe propiedades antioxidantes, antifúngicas contra Candida albicans y Candida tropicalis y antibacteriales contra Staphylococcus aureus y Escherichia colli.

Los resultados obtenidos en esta investigación indican que la dosis fungicida es más baja en comparación con la obtenida por García et al., (2006) quienes evaluaron la actividad antifúngica del A.E. de orégano $(O$. vulgare) sobre el hongo Aspergillus flavus, el cual presentó una inhibición completa con una concentración de $250 \mu \mathrm{g} \mathrm{mL}-1$ y una dosis mínima fungicida de $1.000 \mathrm{\mu g} \mathrm{mL}^{-1}$, demostrando que el aceite de orégano es más eficiente para controlar el patógeno $P$. infestans, frente a otros extractos vegetales, en comparación con esta investigación a $75 \mu \mathrm{g} \mathrm{mL}^{-1}$ se logra inhibir $100 \%$ el crecimiento de $P$. infestans; esto indica que $P$. infestans es más sensible a los compuestos de L. origanoides. Esta diferencia se asocia al alto contenido de timol y otros compuestos fenólicos a los cuales se les atribuye la actividad biocida, los cuales por lo general no están en la misma proporción en las diferentes especies evaluadas. Además, se ha identificado que los fenoles afectan la membrana citoplasmática ocasionando el rompimiento de la fuerza motriz del protón, flujo de electrones, coagulación del contenido celular y acción sobre algunos sistemas enzimáticos involucrados (Davidson, 1997).

\section{Concentración efectiva $50\left(\mathrm{CE}_{50}\right)$}

Las concentraciones evaluadas según se indican la Tabla 4, presenta valores moderadamente altos de inhibición del crecimiento micelial de $P$. infestans en comparación al testigo sin A.E. a medida que disminuye la concentración, también disminuye la actividad biocida. La Tabla 4 muestra el crecimiento micelial de $P$. infestans a frente a diferentes concentraciones del aceite esencial y las figuras 3 y 4 , indican el porcentaje de inhibición de este patógeno. Con estos resultados analizados a través del procedimiento Probit y regresión lineal se estableció que la concentración de aceite esencial de $L$. origanoides capaz de inhibir el crecimiento de $P$. infestans en un $50 \%$ es de $9,29 \mu \mathrm{g} \mathrm{mL}^{-1}$. La CMI junto con la $C_{50}$, constituyeron los referentes para estimar las dosis a aplicar a nivel de campo en la etapa de evaluación del A.E de $L$. origanoides frente a $P$. infestans en cultivo de tomate en invernadero.

\section{Evaluación del A.E. sobre $P$. infestans, ensayo de bioautografía}

La actividad biocida del A.E. de $L$. origanoides frente a $P$. infestans se determinó mediante bioautografía sobre placas cromatográficas de silica gel, en donde se pudo determinar la acción inhibitoria de los compuestos del A.E fraccionados mediante C.C.D, a continuación, se presenta los resultados obtenidos. 


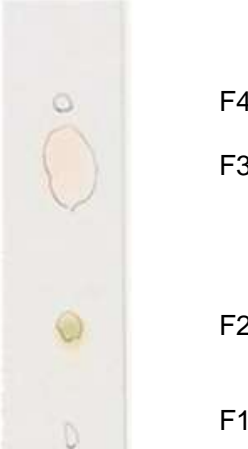

(a) Recorrido del AE

F4

F3

F2

F1 (b) Recorrido estándar timol
F3

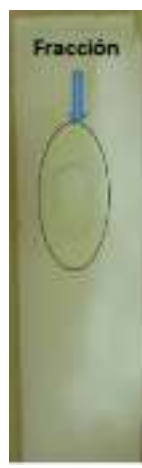

(c) Evaluación por bioautografía

Fig. 5. Resultados de Cromatografía en Capa Delgada y Bioautografía.

La figura $5 a$ muestra el fraccionamiento del A.E. de L. origanoides mediante cromatografía de capa delgada (CCD), de la misma manera la figura $5 \mathrm{~b}$ indica el resultado de la corrida del estándar timol (Sigma Aldrich $\geq$ 99\%) en placas cromatográficas, donde coinciden con la fracción F3 del A.E. La figura $5 c$ por su parte, muestra el bioautograma donde se aprecia la zona de inhibición para $P$. infestans, correspondiente a la fracción F3 compuesto timol, metabolito secundario mayoritario presente en el aceite esencial de orégano silvestre.

El metabolito timol es el responsable de La inhibición de $P$. infestans que se determinó mediante la técnica de bioautografía, acorde con los resultados obtenidos de Rf en CCD y al patrón comercial de timol. El resultado de esta investigación es similar al estudio realizado por Ruano y Figueroa (2013) en el cual afirman que timol es el metabolito secundario mayoritario en el A.E. de L. origanoides H.B.K del Alto Patía y el causante de inhibir el crecimiento de $R$. solani en el bioautograma.

García y Palou (2008) y Acevedo et al. (2013) indican que el timol tiene varios sitios de acción dentro de las células y dependiendo de las concentraciones utilizadas pueden inhibir o inactivar la actividad microbiana. La acción de estos monoterpenos radica en la afección a las membranas y pared celular, enzimas metabólicas, síntesis de proteínas y sistema genético. Por otro lado, el timol y en sí el aceite esencial que lo contiene, posee características de hidrofobicidad, lo cual permite la separación de lípidos de la membrana celular y la mitocondria, desestabilizando la estructura y haciéndola más permeable, ocasionando la filtración de iones y contenidos celulares. Así mismo, se ha identificado que el timol es considerado como uno de los agentes antimicrobiano más activo. Teniendo en cuenta lo anterior, se observa el efecto antimicrobiano que ejercen los compuestos aromáticos (grupos hidroxilo) sobre la membrana citoplasmática provocando su mal funcionamiento. En este caso, el timol une las proteínas hidrofóbicas de la membrana mediante puentes de hidrogeno, cambiando las características de permeabilidad de la misma (Oosterhaven et al., 1995).

Con lo anterior, se puede inferir que el potencial inhibitorio del A.E. de L. origanoides basa su efecto en este compuesto y se soporta en esta investigación en la inhibición micelial de este microorganismo.

\section{CONCLUSIONES}

De acuerdo a los resultados de este estudio, y de su discusión y análisis se pueden extraer las siguientes conclusiones: 1) El aceite esencial (A.E.) de Lippia origanoides H.B.K. ejerció control en el crecimiento del Oomycete Phytophthora infestans aislado de S. lycopersicum; 2) Se identificó como compuesto principal del A.E. de L. origanoides H.B.K. al timol con $83 \%$, siendo este monoterpeno el que tiene efecto inhibitorio sobre $P$. infestans según la prueba de bioautografia. 3) Se demostró el potencial del A.E. de orégano silvestre para el manejo del fitopatógeno y su viabilidad para el desarrollo de un bioinsumo agrícola.

\section{REFERENCIAS}

Álvarez, D., Hurtado, A. y Acosta, J. Aceite esencial de Lippia origanoides como posible producto biorracional en el cultivo de papa (Solanum tuberosum). Vitae. 23 (1), S526 - S530 (2016).

Ahmad, A., A. Khan, F. Akhtar, S. Yousuf, I. Xess, L.A. Khan y N. Manzoor. Fungicidal activity of thymol and carvacrol by disrupting ergosterol biosynthesis and membrane integrity against Candida. https://doi.org/10.1007/s10096-010-1050-8. European Journal of Clinical Microbiology and Infectious Diseases, 30(1), 41-50. (2011).

Arango, O., A.M. Hurtado, y I. Toro. Effect of origin, harvest time and leaves age on the yield and thymol content of essential oils from Lippia origanoides H.B.K. Acta Agronomica, 61(3), 207-213. (2012).

Arango, O., A. Hurtado, D. Pantoja, y L. Santacruz. Actividad inhibitoria del aceite esencial de Lippia origanoides H.B.K sobre el crecimiento de Phytophthora infestans. https://doi.org/10.15446/acag.v64n2.42964. Acta Agronomica, 64(2). (2014). 
Acevedo, D., Navarro, M. y Monroy, L. Composición Química del Aceite Esencial de Hojas de Orégano (Origanum vulgare). Información Tecnológica, 24 (4), 43 - 48. (2013).

Bueno-Sanchez, J. G., E.D. Coy, y E.E. Stashenko. Antimycobacterial natural products--an opportunity for the Colombian biodiversity. Revista Española de Quimioterapia: Publicación Oficial de La Sociedad Española de Quimioterapia, 24(4), 175-183. (2011).

Celis, C., P. Escobar, J. Isaza, E. Stashenko y J. Martínez. Estudio comparativo de la composición y actividad biológica de los aceites esenciales extraídos de Lippia alba, Lippia origanoides y Phyla dulcis, especies de la familia Verbenaceae. Scienctia et Technica, (33), 103-105. (2007).

Davidson, P. Chemial preservatives and natural antimicrobial compounds. En: M. P. Doyle, L.R. Beuchat y T.J. Montville (Eds). Food Microbiology - Fundamentals and Frontiers. ASM Press. Washington D.C. pp. 520-556. (1997).

Dos Santos, F., J. Lopes y otros autores. Composition and biological activity of essential oils from Lippia origanoides H.B.K. Essen Oil Res, 16, 504-506. (2004).

Escobar, P., S.M. Leal, L.V. Herrera, J.R. Martinez y E. Stashenko. Chemical composition and antiprotozoal activities of Colombian Lippia spp essential oils and their major components. https://doi.org/10.1590/S0074-02762010000200013. Memorias Do Instituto Oswaldo Cruz, 105(2), 184-190. (2010).

García, C., A. Martínez, J. Ortega, y F. Castro. Componentes químicos y su relación con las actividades biológicas de algunos extractos vegetales. Revista QuímicaViva, 2, 86-96. (2010).

García, E.A., M.Y. Quezada, J. Moreno, G. Sánchez, E. Moreno, M.J. Pérez. Actividad Antifúngica de Aceites Esenciales de Canela (Cinnamomum zeylanicum Blume) y Orégano (Origanum vulgare L.) y su Efecto sobre la Producción de Aflatoxinas en Nuez Pecanera [Carya illinoensis (F.A. Wangenh) K. Koch]. Revista Mexicana de Fitopatología, vol. 24, núm. 1, enero-junio, pp. 8-12. (2006).

García, R.M. y E. Palou. Mecanismos de accion antimicrobiana de timol y carvacrol sobre microorganismos de interés en alimentos. Temas Selectos de Ingeniería de Alimentos. 2-2 p 41-51. (2008)

Kintzios, E. Orégano. The genera Origanum and Lippia. London and New York: Taylor \& Francis. 267 p. (2002).

Krolow, V., C. Bauer y J. Costa. Efeito de óleos essenciais de plantas medicinais no controle de Phytophthora infestans em batata. Resumos do I Congresso Brasileiro de Agroecologia. Ver. Brasil. Agroec. 1(1):1712 - 1724. (2006.).

Martínez, F. E., C.E. Aíl-catzím, L.G. Hernández-montiel, C. Lizette, D. Toro, \& E.O. Rueda-puente. Hongos Fitopatógenos Asociados Al Tomate (Solanum Lycopersicum L.) En La Zona Árida Del Noroeste De México: La Importancia De Su Diagnóstico. https://doi.org/10.19044/esj.2016.v12n18p232. European Scientific Journal, 12(18), 232-256. (2016).

Muñoz-Acevedo, A., J.R. Martínez y E.E. Stashenko. Cromatografía de gases como herramienta de estudio de la composición química y capacidad antioxidante de especies vegetales ricas en timol y carvacrol, cultivadas en Colombia. Scientia Chromatographica, 67-78. (2009).

Oosterhaven, K., B. Poolman y E. Smid. S-carvone as a natural potato sprout inhibiting, fugistatic ans bacteristatic compound.Industrial Crops and Products. 4. 23-31. (1995).

Riveros, B., R. Sotomayor, V. Rivera, V. Secor, y B. Espinoza. Resistencia de Phytophthora infestans (Montagne) de Bary a metalaxil, en cultivo de papas en el norte de Chile. Agric. Téc., 63, 2. (2003).

Ruano, O y L. Figueroa. Evaluación de la sensibilidad del patógeno Rhizoctonia solani aislado de papa (Solanum tuberosum L.) frente al aceite esencial de orégano silvestre Lippia origanoides H.B.K, y determinación de la fraccion mas activa a nivel in vitro. Tesis. Facultad de Agroindustria. Universidad de Nariño. (2013).

Shattock, R. Studies on the inheritance of resistance to Metalaxyl in Phytophthora infestans. (P. Pathology, Ed.). p.67 (1998).

Sridhar, S., Rajagopal, R., Rajavel, R., y Masilamani, S. Narasimhan, S. Antifungal Activity of Some Essential Oils. Journal of Agricultural Food Chemistry, 51, 7596-7599. (2003).

Stashenko, E. E., y J.R. Martínez. Algunos aspectos prácticos para la identificación de analitos por cromatografía de gases acoplada a espectrometría de masas. Scientia Chromatographica, 2(1), 29-47. (2010)

Torrenegra, M. E. Evaluación de la actividad antioxidante del aceite esencial foliar extraído de especies de oregano (Origanum vulgare), oregano "borde blanco" (Origanum vulgare ssp) y oreganito (Lippia alba mill) cultivado en la zona norte del departamento de Bolívar. Tesis de Magíster. Dpto de Ingeniería Agrícola y de Aimentos. Universidad Nacional de Colombia p.131. (2014).

Trombetta, D, C., Sarpietro, M.G, Venuti, V, Cristani, M, Daniele, C, Saija A, Mazzanti G, Bisignano G. Mechanisms of antibacterial action of three monoterpenes. Antimicrob Agents Chemother. 49(6):2474-8. (2005)

Vásquez, D. El orégano de monte (Lippia origanoides) del Alto Patía: Efecto del método de obtención de sus extractos sobre la composición y la actividad antioxidante de los mismos. Tesis de Magíster. Dpto. de química. Universidad Nacional de Colombia. p. 123. (2012).

Vicuña, G. C., E.E. Stashenko y J.L. Fuentes. Chemical composition of the Lippia origanoides essential oils and their antigenotoxicity against bleomycin-induced DNA damage. Fitoterapia, 81(5), 343-349. (2010). 